\title{
Dispositivo estructurado de intervenciones psicosociales en atención primaria: serie de casos retrospectiva
}

\author{
Structured psychosocial interventions in primary care: retrospective case series
}

Cecilia Drimer ${ }^{\mathrm{a}}$, Sergio Terrasa ${ }^{\mathrm{a}}$, Mercedes Mutchinick $^{\mathrm{a}}$

\begin{abstract}
Resumen
Antecedentes: Más allá del pago por cápita, desde 2009 el Plan de Salud del Hospital Italiano de Buenos Aires reconoció a los médicos de familia el pago por prestación de intervenciones psicosociales de cuarenta minutos de duración realizadas para promover el bienestar y la autonomía de sus pacientes.

Objetivos: Describir los problemas que motivaron estas intervenciones y las redefiniciones diagnósticas que realizaron estos profesionales.

Métodos: Fueron revisadas las fichas estructuradas de registro de 482 intervenciones psicosociales realizadas durante 2011 y codificadas mediante la Clasificación Internacional de la Atención Primaria (CIAP-2).

Resultados: Los motivos de consulta más frecuentes fueron los sentimientos depresivos y/o de ansiedad (33,25\%), problemas familiares y/o vinculados a crisis vitales $(16 \%)$, dolor $(9,56 \%)$ y cansancio $(2,91 \%)$. Entre las redefiniciones diagnósticas predominaron las crisis vitales (15,45\%), los problemas de la relación conyugal o con hijos (14,61\%), y los trastornos depresivos y/o de ansiedad (27\%).

Conclusiones: nuestro modelo de trabajo contribuyó a que en una gran proporción de pacientes que había consultado por dolor u otros síntomas generales, detectáramos, abordáramos y documentáramos el proceso de atención de problemas de la esfera psicosocial, que suele ser subregistrado con el abordaje biomédico clásico.
\end{abstract}

\begin{abstract}
Background: Beyond capitation payment, since 2009 Hospital Italiano de Buenos Aires Health Maintenance Organization incorporated "structured primary care psychosocial interventions" as a fee for service practice. They last 40 minutes and are undertaken by family physicians with the aim of improving the wellbeing of their patients and helping them to strengthening their autonomy.

Objectives: To identify chief complaints and problems (re)definitions carried out by family physicians.

Methodology: 482 medical records written during 2011 were reviewed and coded according to the International Classification of Primary Care (ICPC-2).

Results: Most frequent chief complaints were depressive and/or anxious feelings (33.25\%), family problems and/or phases of adult life problems (16\%), pain $(9.56 \%)$ and fatigue $(2.91 \%)$. Most common problem (re)definitions were life events $(15.45 \%)$, followed by marital or child-related problems (14.61\%), and depressive and/or anxiety disorders $(27 \%)$.

Conclusions: Our working model enabled us to identify, address and document psychosocial problems -which are often underreported within the classical biomedical approach- in a large proportion of patients whose chief complaint were pain or other general symptoms.
\end{abstract}

Drimer C, Terrasa S, Mutchinick M.. Dispositivo estructurado de intervenciones psicosociales en atención primaria: serie de casos retrospectiva. Evid Actual Pract Ambul. 2019;22(2):e002014.

\section{Introducción}

La Medicina Familiar es una especialidad horizontal que procura abordar los problemas de sus pacientes en el contexto de su situación vital, dentro de un marco familiar, e integrando ciencias biológicas, clínicas y de la conducta ${ }^{1-3}$.

Además, el médico de familia puede, en algunas ocasiones, ofrecer a sus pacientes y/o familias dispositivos de "atención psicosocial ampliada" que, en la bibliografía, suelen ser mencionados como intervenciones psicosociales en atención primaria ${ }^{4}$. Como fue documentado por Huibers MJH en su revisión sistemática, la evidencia de la eficacia de este tipo de intervenciones es todavía escasa debido a las limitaciones metodológicas de las investigaciones que las evaluaron ${ }^{5,6}$ y a que es difícil realizar comparaciones experimentales entre los distintos dispositivos existentes.

En el Servicio de Medicina Familiar y Comunitaria del Hospital Italiano de Buenos Aires -Hospital Universitario Privado que atiende predominantemente población urbana del Área Metropolitana de Buenos Aires-, hemos desarrollado una herramienta para sistematizar el registro de las intervenciones psicosociales que llevan a cabo los médicos de familia en su práctica cotidiana, basándose en una modalidad de atención denominada FOCO (Familia, Orientación y COntexto) a través de sucesivas entrevistas prolongadas, con una duración estimada de 40 minutos (las habituales duran 20 minutos) con su médico de familia.

En la ficha estructurada de registro (ver Appendix ) el profesional debe describir el motivo de la consulta y el contexto en el que ésta está ocurriendo, su reformulación del problema o diagnóstico contextual operativo (DCO), la motivación del paciente o la familia para trabajar en dicho dispositivo, los objetivos del equipo terapéutico y el plan para la entrevista siguiente ${ }^{7}$.

En las entrevistas del dispositivo FOCO, el médico de familia que actúa como médico de cabecera del paciente, realiza intervenciones individuales, de pareja y/o de familia con el objetivo de movilizar los recursos del paciente y/o su grupo familiar y contribuir al desarrollo de estrategias personalizadas para aliviar su

\footnotetext{
a Servicio de Medicina Familiar y Comunitaria, Hospital Italiano de Buenos Aires. cecilia.drimer@hospitalitaliano.org.ar
} 
padecer y/o para optimizar el desempeño de su sistema de cuidados.

Son candidatos a ser atendidos mediante el dispositivo FOCO aquellos pacientes/familias que requieren un tiempo y una atención específica para que el médico de familia se dedique a orientarlos con relación a cambios en el estilo de vida, al abordaje de ciertas enfermedades crónicas, al manejo de situaciones agudas que modifican la vida cotidiana, situaciones de duelo, estrés del cuidador, disfunción familiar, crisis vitales, problemas psicosociales como ansiedad o depresión y todo problema contextual que requiera un mayor detenimiento. De este modo, el médico de familia intenta ayudar en el proceso de cambio del paciente y de su familia en relación con los problemas citados y procura ayudar o acompañar en los procesos de toma de decisiones, con el fin último de que este sistema (paciente y su familia) logre la mayor autonomía posible en su cuidado (autocuidado).

Este dispositivo debe tener un foco de trabajo claro y definido, $y$ su duración debe ser limitada en el tiempo (habitualmente desarrollado entre una y seis entrevistas), dado que no se pretende que funcione como una psicoterapia o consejería de tiempo indefinido ${ }^{7}$

La tarea desarrollada mediante el dispositivo FOCO es supervisada mediante diferentes instancias. Entre ellas citamos a las Unidades Docentes Asistenciales (espacio de supervisión por pares, integrado por profesionales de distinta experiencia: médicos de planta, médicos asociados y residentes, que se reúnen semanalmente), la supervisión individual (sobre todo en aquellos casos en los que el médico interviniente lo considere necesario) y los ateneos de FOCO, que tienen realización periódica.

El Hospital Italiano de Buenos Aires cuenta con un Seguro de Salud que durante 2009 reconoció institucionalmente dicha modalidad de trabajo de su Servicio de Medicina Familiar y Comunitaria, lo cual implicó además el reconocimiento económico de dicha práctica, equivalente al tiempo adicional empleado durante las intervenciones psicosociales en atención primaria cuando se compara su duración con la de una entrevista habitual (aproximadamente el doble de tiempo).

Desde ese año y hasta la fecha, se realizaron en el Servicio de Medicina Familiar y Comunitaria un número creciente de entrevistas de FOCO y cada vez más especialistas en Medicina Familiar utilizan este dispositivo. Fue en este contexto que surgió la inquietud de nuestro grupo médico de conocer las características de las entrevistas, así como el perfil de los médicos y los pacientes intervinientes. Nos planteamos entonces como objetivos principales describir la prevalencia de los motivos de consulta de las entrevistas, así como las reformulaciones diagnósticas realizadas durante 2011. Los objetivos secundarios fueron describir el perfil demográfico de los pacientes/familias y médicos que participaron de este dispositivo, conocer la proporción de entrevistas que fueron de primera vez y cuáles de seguimiento, así como la proporción de tratamientos que el profesional interviniente consideró concluidos, y, además, evaluar la calidad del registro de dichas consultas mediante el uso de la ficha estructurada creada a tal fin.

\section{Métodos}

El diseño del trabajo fue desarrollado con el formato de una serie histórica de casos.

La población estudiada correspondió a una muestra de 482 consultas de afiliados al Plan de Salud del Hospital Italiano de Buenos Aires que tenían como médico de cabecera a un médico de familia perteneciente al Servicio de Medicina Familiar y Comunitaria y cuya primera consulta con el dispositivo FOCO sucedió durante 2011
Los motivos de consulta y las reformulaciones diagnósticas 0 DCO fueron clasificados sobre la base de las 17 categorías de la versión española de la Segunda edición de la Clasificación Internacional de la Atención Primaria de la Organización Mundial de los Médicos Generales/de Familia (CIAP-2) ${ }^{8}$.

A los efectos de esta investigación, se consideró que el registro de cada consulta fue adecuado cuando los investigadores pudieron constatar que estaba consignada la información de las siguientes cuatro categorías: 1) el motivo de consulta del paciente, 2) la relación con el contexto, 3) la redefinición del problema a través del DCO, 4) el objetivo de trabajo que se llevaría a cabo a través de una estrategia determinada (incluso en aquellos casos en los que no se haya respetado estrictamente el orden de las consignas).

Nuestra investigación fue realizada con datos obtenidos a través de la historia clínica electrónica (HCE). Se solicitó al Servicio de Informática Médica del HIBA una base de datos que contenía los 996 registros de las entrevistas realizadas en el marco del dispositivo FOCO durante 2011 , de las cuales fueron seleccionadas en forma aleatoria 482 para ser revisadas manualmente.

\section{Procedimientos para garantizar los aspectos éticos de la investigación}

Con el objetivo de revisar la información previamente detallada, la investigadora principal accedió a una lista que incluyó a los 482 registros clínicos seleccionados en forma aleatoria, que no contenía información que permitiera identificar al paciente. Dado que fue garantizado el manejo anónimo de los datos, no se solicitó consentimiento a los participantes. El protocolo fue aprobado con el Nro. 1883 por el Comité de Ética de Protocolos del Hospital Italiano de Buenos Aires.

\section{Resultados}

Se presentan los resultados de una serie de 482 consultas en las que se utilizó el dispositivo FOCO realizadas durante 2011, que representan el $48 \%$ del total $(n=996)$ de las realizadas durante ese año.

Estas 482 consultas correspondieron a un total de 286 pacientes. Las características de los pacientes y de los profesionales que participaron fueron resumidas en la Tabla 1 y la Tabla 2. De ellas se desprende que el $68 \%$ de las entrevistas fueron realizadas por médicos de 40 a 49 años que en su mayoría eran médicos de planta. Los pacientes fueron predominantemente mujeres mayores de 30 años y, en alrededor de la mitad de los casos, al menos algún otro miembro de su familia había sido atendido por el mismo médico como médico de cabecera.

Con el objetivo de obtener una interpretación acerca de la continuidad de la intervención, se confeccionó la Tabla 3, en la cual podemos observar cómo el $58 \%$ de los pacientes realizó solo una entrevista, $24 \%$ dos entrevistas, $12 \%$ tres entrevistas y el $6 \%$ restante, cuatro o más. De todas formas, solo en un pequeño porcentaje de los registros fue explicitada la conclusión de la intervención.

En relación a la calidad de registro, más del $90 \%$ de las consultas tuvieron registrado adecuadamente el motivo de consulta y la descripción contextual del paciente, mientras que en el $76 \%$ de los registros figuraba el DCO y sólo en $58 \%$, el objetivo de la implementación del dispositivo FOCO.

Para poder analizar la distribución del total de las entrevistas según su motivo de consulta y la reformulación hacia el DCO consignado, confeccionamos la Tabla 4. En esta se muestra en cuántas consultas fue registrado cada uno de los motivos mencionados y su reformulación o DCO. Es decir que, si bien inicialmente cierto número de pacientes consultó por un motivo determinado, 
el DCO final pudo ser diferente en cada uno de ellos. Por ejemplo, podemos observar que 50 pacientes consultaron por dolor o síntomas generales, pero solo en siete pacientes se estableció el mismo DCO, ya que en el resto el diagnóstico fue reformulado a algún otro DCO. Por otro lado, hubo 77 consultas motivadas por crisis vitales $u$ otros problemas familiares, que luego subieron a 161 luego de la reformulación diagnóstica de otros que habían consultado por algún otro motivo aparente.

A partir de la información resumida en la \$ se confeccionaron la Tabla 5 y laTabla 6, que describen en más detalle los ítems más frecuentes, categorizados mediante CIAP-2 y la denominación que usamos habitualmente en nuestra práctica clínica. nterpretando los datos obtenidos, podemos ver que los motivos de consulta más frecuentes fueron los "sentimientos y/o los trastornos depresivos $y / 0$ de ansiedad", los problemas vinculados con las "crisis vitales o problemas de la relación conyugal o con hijos" y el "dolor, cansancio o síntomas generales". Y al realizar la reformulación diagnóstica, predominaron las "crisis vitales o problemas de la relación conyugal o con los hijos", seguidas por los "sentimientos o trastornos depresivos y/o de ansiedad".

\section{Discusión}

Como era nuestra presunción, una gran proporción de los problemas que motivaron la consulta de los pacientes relacionados al dolor u otros síntomas generales fueron reformulados como problemas de la esfera psicosocial, como son las crisis vitales y/o los problemas de la relación conyugal o con los hijos. Consideramos que esta forma de abordaje amplía las herramientas disponibles para ayudar a nuestros pacientes, enfocando la terapéutica a sus verdaderas necesidades y evitando en muchos casos, exámenes complementarios y derivaciones potencialmente innecesarios.

Tabla 1. Características de la población atendida atendida a través del dispositivo FOCO (Familia, Orientación y COntexto), en un Servicio de Medicina Familiar de un Hospital Universitario.

\begin{tabular}{|l|l|c|}
\hline \multicolumn{2}{|c|}{ Pacientes (n=482) } & $\begin{array}{c}\text { Número de } \\
\text { entrevistas } \\
(\%)\end{array}$ \\
\hline \multirow{3}{*}{$\begin{array}{l}\text { Franjas } \\
\text { de } \\
\text { edad } \\
\text { (años) }\end{array}$} & Menor de 21 & $13(2,7)$ \\
\cline { 2 - 3 } & 21 a 29 & $20(4,15)$ \\
\cline { 2 - 3 } & 30 a 39 & $85(17,63)$ \\
\cline { 2 - 3 } & 40 a 49 & $80(16,60)$ \\
\cline { 2 - 3 } & 50 a 59 & $83(17,22)$ \\
\cline { 2 - 3 } & 60 a 69 & $111(23,03)$ \\
\cline { 2 - 3 } & 70 o mayor & $90(18,67)$ \\
\hline \multirow{2}{*}{$\begin{array}{l}\text { Sexo femenino } \\
\text { Al menos un familiar también atendido habitualmente } \\
\text { por ese médico de familia }\end{array}$} & $248(51,45)$ \\
\hline
\end{tabular}

Tabla 2. Características de los médicos que realizaron entrevistas de FOCO (Familia, Orientación y COntexto).

\begin{tabular}{|c|c|c|}
\hline \multicolumn{2}{|r|}{ Médicos $(n=482)$} & Número de \\
\hline \multirow{5}{*}{$\begin{array}{l}\text { Franjas } \\
\text { de } \\
\text { edad } \\
\text { (años) }\end{array}$} & Menor de 35 & $49(10,17)$ \\
\hline & 35 a 39 & $83(17,22)$ \\
\hline & 40 a 44 & $171(35,48)$ \\
\hline & 45 a 49 & $159(32,78)$ \\
\hline & 50 o mayor & $21(4,36)$ \\
\hline \multicolumn{2}{|c|}{ Sexo Femenino } & $239(49,59)$ \\
\hline \multirow[t]{3}{*}{ Puesto } & Residente & 0 \\
\hline & Asociado & $153(31,88)$ \\
\hline & Planta & $327(68,13)$ \\
\hline
\end{tabular}

Tabla 3. Distribución de las fichas de registro de las consultas FOCO (Familia, Orientación y COntexto) de acuerdo al tipo de entrevista, la motivación consignada en la misma y la calidad de su registro

\begin{tabular}{|c|c|c|}
\hline \multicolumn{2}{|c|}{ Características de las fichas $(\mathrm{n}=482)$} & \multirow{2}{*}{$\begin{array}{c}\begin{array}{r}\text { Número de } \\
\text { entrevistas } \\
(\%)\end{array} \\
232(48,13)\end{array}$} \\
\hline \multirow[t]{3}{*}{ Tipo de ficha } & Inicial & \\
\hline & Seguimiento & $220(45,64)$ \\
\hline & Indeterminado & $30(6,22)$ \\
\hline \multirow[t]{4}{*}{ Motivación } & De ambos & $98(20,33)$ \\
\hline & Del médico & $121(25,10)$ \\
\hline & Del paciente & $145(30,08)$ \\
\hline & Indeterminado & $118(24,48)$ \\
\hline \multirow{4}{*}{$\begin{array}{l}\text { Documentación } \\
\text { adecuada en } \\
\text { la ficha de } \\
\text { registro }\end{array}$} & Del motivo de consulta & $444(92,12)$ \\
\hline & Del contexto & $439(91,08)$ \\
\hline & $\begin{array}{l}\text { Del diagnóstico contextual opera- } \\
\text { tivo }\end{array}$ & $369(76,56)$ \\
\hline & Del objetivo de las entrevistas & $280(58,09)$ \\
\hline
\end{tabular}


Tabla 4. Distribución del total de las consultas registradas de acuerdo al motivo de consulta y al diagnóstico contextual operativo codificado por la Clasificación Internacional de Atención Primaria (CIAP-2) y la denominación usual que se le da nuestra práctica habitual.

\begin{tabular}{|c|c|c|c|}
\hline Denominación usual & $\begin{array}{l}\text { Denominación de la Clasificación Internacional } \\
\text { de Atención Primaria (CIAP-2) }\end{array}$ & $\begin{array}{l}\text { Motivo de consulta } \\
\qquad \mathrm{N}(\%)\end{array}$ & $\begin{array}{c}\text { Diagnóstico } \\
\text { contextual } \\
\text { operativo } \\
\mathrm{N}(\%)\end{array}$ \\
\hline Cansancio & Neurastenia/surmenage & $14(2,91)$ & $7(1,46)$ \\
\hline \multirow{2}{*}{$\begin{array}{l}\text { Trastorno de la conducta } \\
\text { alimentaria }\end{array}$} & Anorexia, bulimia & $4(0,83)$ & $1(0,21)$ \\
\hline & Problemas de la conducta alimentaria en niños & $1(0,21)$ & $1(0,21)$ \\
\hline $\begin{array}{l}\text { Preocupación por la aparien- } \\
\text { cia }\end{array}$ & Preocupación por la apariencia & $1(0,21)$ & $2(0,42)$ \\
\hline \multirow{4}{*}{$\begin{array}{l}\text { Dolor y otros síntomas genera- } \\
\text { les }\end{array}$} & Dolor generalizado/dolores múltiples & $19(3,95)$ & $3(0,63)$ \\
\hline & Dolor de pecho & $3(0,62)$ & 0 \\
\hline & Otros signos/síntomas generales. & $27(5,61)$ & $4(0,84)$ \\
\hline & Sensación de estar enfermo & $1(0,21)$ & 0 \\
\hline \multirow{2}{*}{ Enfermedades generales } & Cáncer & $2(0,42)$ & 0 \\
\hline & Otras enfermedades generales & $8(1,66)$ & $2(0,42)$ \\
\hline \multirow[t]{4}{*}{ Abuso de sustancias } & Abuso agudo del alcohol & $2(0,42)$ & $1(0,21)$ \\
\hline & Abuso crónico del alcohol & $5(1,04)$ & $6(1,25)$ \\
\hline & Abuso de drogas & $4(1,04)$ & $6(1,25)$ \\
\hline & Abuso de tabaco & $6(1,25)$ & $4(0,84)$ \\
\hline Demencia & Demencia & $1(0,21)$ & $1(0,21)$ \\
\hline \multirow[t]{4}{*}{ Preocupación o miedo } & Preocupación o miedo a la muerte/agonía & $2(0,42)$ & 0 \\
\hline & Preocupación o miedo a otra enfermedad & $12(2,49)$ & $5(1,04)$ \\
\hline & Preocupación o miedo a cáncer & $4(0,83)$ & 0 \\
\hline & Preocupación o miedo al tratamiento & $3(0,62)$ & 0 \\
\hline Violencia o agresividad & Violencia o agresividad & $6(1,25)$ & $6(1,25)$ \\
\hline \multirow[t]{4}{*}{ Problemas sociales } & Problema laboral & $7(1,46)$ & $5(1,04)$ \\
\hline & Problema legal & 0 & $1(0,21)$ \\
\hline & Problema sociocultural & 0 & $2(0,42)$ \\
\hline & Otros problemas sociales & $4(0,83)$ & $2(0,42)$ \\
\hline
\end{tabular}




\begin{tabular}{|c|c|c|c|}
\hline Denominación usual & $\begin{array}{c}\text { Denominación de la Clasificación Internacional } \\
\text { de Atención Primaria (CIAP-2) }\end{array}$ & $\begin{array}{l}\text { Motivo de consulta } \\
\text { N (\%) }\end{array}$ & $\begin{array}{c}\text { Diagnóstico } \\
\text { contextual } \\
\text { operativo } \\
\mathrm{N}(\%) \\
\end{array}$ \\
\hline & Miedo a problema social & $3(0,62)$ & 0 \\
\hline \multirow[t]{3}{*}{$\begin{array}{l}\text { Crisis del cuidador o } \\
\text { preocupación por enfermedad } \\
\text { de allegado }\end{array}$} & $\begin{array}{l}\text { Crisis del cuidador o preocupación por enferme- } \\
\text { dad de los hijos }\end{array}$ & $15(3,12)$ & $14(2,92)$ \\
\hline & $\begin{array}{l}\text { Crisis del cuidador o preocupación por enferme- } \\
\text { dad del cónyuge }\end{array}$ & $8(1,66)$ & $11(2,3)$ \\
\hline & $\begin{array}{l}\text { Crisis del cuidador o preocupación por enferme- } \\
\text { dad del padre u otros familiares }\end{array}$ & $10(2,08)$ & $10(2,09)$ \\
\hline \multirow{8}{*}{$\begin{array}{l}\text { Crisis vital u otros problemas } \\
\text { familiares } \\
\text { (ej. de pareja, } \\
\text { de crianza) }\end{array}$} & $\begin{array}{l}\text { Queja o problema por el comportamiento o con- } \\
\text { ducta del niño }\end{array}$ & $2(0,42)$ & 0 \\
\hline & $\begin{array}{l}\text { Queja o problema por el comportamiento o con- } \\
\text { ducta del adolescente }\end{array}$ & $1(0,21)$ & $1(0,21)$ \\
\hline & $\begin{array}{l}\text { Queja o problema por el comportamiento o con- } \\
\text { ducta del cónyuge }\end{array}$ & $8(1,66)$ & $6(1,25)$ \\
\hline & $\begin{array}{l}\text { Queja o problema por el comportamiento o con- } \\
\text { ducta de los padres u otros familiares }\end{array}$ & $4(0,83)$ & $2(0,42)$ \\
\hline & Problemas en la relación con los hijos & $15(3,12)$ & $23(4,8)$ \\
\hline & Problemas en la relación conyugal & $38(7,90)$ & $47(9,81)$ \\
\hline & $\begin{array}{l}\text { Problemas en la relación con los padres u otros } \\
\text { familiares }\end{array}$ & $5(1,04)$ & $8(1,67)$ \\
\hline & Problemas en etapa de la vida adulta & $24(4,99)$ & $74(15,45)$ \\
\hline \multirow[t]{3}{*}{ Duelo por pérdida o muerte } & $\begin{array}{l}\text { Duelo por pérdida o muerte de padres o familia- } \\
\text { res }\end{array}$ & $9(1,87)$ & $18(3,76)$ \\
\hline & Duelo por pérdida o muerte de hijo & $5(1,04)$ & $10(2,09)$ \\
\hline & Duelo por pérdida o muerte de cónyuge & $5(1,04)$ & $7(1,46)$ \\
\hline \multirow[t]{5}{*}{ Ansiedad } & Sentimientos de ansiedad o tensión & $69(14,35)$ & $27(5,64)$ \\
\hline & Sentimientos de irritabilidad o enojo & $8(1,66)$ & $8(1,67)$ \\
\hline & Trastorno de ansiedad & $6(1,25)$ & $34(7,10)$ \\
\hline & Trastorno compulsivo/Fobia & 0 & $1(0,21)$ \\
\hline & Trastorno de estrés post traumático & 0 & $8(1,67)$ \\
\hline \multirow[t]{2}{*}{ Depresión } & Sentimientos depresivos & $87(18,09)$ & $12(2,51)$ \\
\hline & Trastorno depresivo & 0 & $69(14,41)$ \\
\hline Trastorno de personalidad & Trastorno de personalidad & 0 & $5(1,04)$ \\
\hline
\end{tabular}




\begin{tabular}{|l|l|c|c|}
\hline \multicolumn{1}{|c|}{ Table 4 continued } & $\begin{array}{c}\text { Denominación de la Clasificación Internacional } \\
\text { de Atención Primaria (CIAP-2) }\end{array}$ & $\begin{array}{c}\text { Motivo de consulta } \\
\text { N (\%) }\end{array}$ & $\begin{array}{c}\text { Diagnóstico } \\
\text { contextual } \\
\text { operativo } \\
\text { N (\%) }\end{array}$ \\
\hline $\begin{array}{l}\text { Trastorno de somatización/- } \\
\text { conversión }\end{array}$ & Trastorno de somatización/conversión & 0 & $5(1,04)$ \\
\hline $\begin{array}{l}\text { Depresión psicótica o en } \\
\text { paciente con psicosis }\end{array}$ & Psicosis afectiva & 0 & $1(0,21)$ \\
\hline $\begin{array}{l}\text { Otros trastornos de la salud } \\
\text { mental }\end{array}$ & Signos síntomas psicológicos/mentales & $8(1,66)$ & $7(1,46)$ \\
\cline { 2 - 4 } & Otros trastornos psicológicos & $2(0,42)$ & $6(1,25)$ \\
\hline \multirow{2}{*}{ Trastornos del sueño } & Trastornos del sueño & $7(1,46)$ & $4(0,84)$ \\
\hline \multirow{2}{*}{ Problemas de la sexualidad } & Preocupación por las preferencias sexuales & $2(0,42)$ & 0 \\
\cline { 2 - 4 } & Disminución del deseo sexual & $3(0,62)$ & $2(0,42)$ \\
\hline
\end{tabular}

Tabla 5. Distribución de las consultas registradas de acuerdo al motivo de consulta y el diagnóstico contextual operativo más frecuentes, codificadas por la Clasificación Internacional de Atención Primaria (CIAP-2)

\begin{tabular}{|c|c|c|}
\hline Denomiación mediante el sistema CIAP-2 & $\begin{array}{c}\text { Motivo de consulta } \\
\mathrm{N}(\%)\end{array}$ & $\begin{array}{c}\text { Diagnóstico contextual } \\
\text { operativo } \\
\mathrm{N}(\%)\end{array}$ \\
\hline Problemas en la relación conyugal & $38(7,90)$ & $47(9,81)$ \\
\hline Problemas en la relación con los hijos & $15(3,12)$ & $23(4,8)$ \\
\hline Problemas en etapa de la vida adulta (crisis vital) & $24(4,99)$ & $74(15,45)$ \\
\hline Sentimientos depresivos & $87(18,09)$ & 0 \\
\hline Trastorno depresivo & 0 & $69(14,41)$ \\
\hline Sentimientos de ansiedad o tensión & $69(14,35)$ & $27(5,64)$ \\
\hline Trastorno de ansiedad & 0 & $34(7,10)$ \\
\hline Dolor generalizado/múltiple y otros sígno/síntomas generales & $46(9,56)$ & $7(1,47)$ \\
\hline Neurastenia/surmenage (cansancio) & $14(2,91)$ & $7(1,46)$ \\
\hline Estrés del cuidador y/o problemas por enfermedad de los hijos & $15(3,12)$ & $14(2,92)$ \\
\hline Estrés del cuidador y/o problemas por enfermedad de algún progenitor $\mathrm{u}$ otros familiares & $10(2,08)$ & $10(2,09)$ \\
\hline Preocupación o miedo a muerte o enfermedad & $12(2,49)$ & $5(1,04)$ \\
\hline Duelo (pérdida o muerte) de padres o familiares & $9(1,87)$ & $18(3,76)$ \\
\hline Duelo (pérdida o muerte) de hijos & $5(1,04)$ & $10(2,09)$ \\
\hline
\end{tabular}


Tabla 6. Distribución las consultas registradas de acuerdo al motivo de consulta y el diagnóstico contextual operativo más frecuentes, codificadas por la Clasificación Internacional de Atención Primaria (CIAP-2) y la denominación usual que se le da en nuestra práctica habitual

\begin{tabular}{|l|c|c|}
\hline Denominación habitual & $\begin{array}{c}\text { Motivo de consulta } \\
\mathrm{N}(\%)\end{array}$ & $\begin{array}{c}\text { Diagnóstico contextual operativo } \\
\mathrm{N}(\%)\end{array}$ \\
\hline Crisis vital o problemas de la relación conyugal o con hijos & $77(16,01)$ & $144(30,06)$ \\
\hline Sentimientos depresivos & $87(18,09)$ & $69(14,41)$ \\
\hline Sentimientos de ansiedad o tensión & $69(14,35)$ & $61(12,64)$ \\
\hline Dolor, cansancio u otros síntomas generales & $60(12,47)$ & $14(2,93)$ \\
\hline Estrés del cuidador y/o problemas por enfermedad de allegados & $25(5,05)$ & $24(5,01)$ \\
\hline Preocupación o miedo a muerte o enfermedad & $12(2,49)$ & $5(1,04)$ \\
\hline Duelo (pérdida o muerte) & $14(2,91)$ & $28(5,85)$ \\
\hline
\end{tabular}

Entre las debilidades de nuestra investigación cabe destacar que la CIAP-2 fue creada para registrar la actividad realizada en Atención Primaria (razón de la consulta, problema atendido y proceso de atención) y describirla globalmente. Si bien tiene la ventaja de que permite realizar comparaciones entre diferentes instituciones o regiones, o bien entre diferentes actores que trabajan bajo distintos paradigmas de abordaje de los problemas de salud, consideramos que resulta algo rígida como para capturar el espectro de motivos de consulta y de DCO de nuestra práctica cotidiana. En muchos casos, algunos de los términos y agrupaciones diagnósticas que propone la CIAP-2 no coinciden enteramente con la terminología que usamos en nuestra práctica habitual, como lo muestra la Tabla 4. Además, no podemos olvidar que nuestro trabajo refleja exclusivamente lo documentado en la HCE, que puede no ser un fiel reflejo de lo ocurrido en la consulta médica.
Nuestros hallazgos permitieron documentar algunos procesos y productos del trabajo médico en la esfera psico-socio-contextual de nuestros pacientes y familias, que suelen pasar inadvertidos cuando se los evalúa a través de indicadores de utilización (p. ej. proporción de turnos libres respecto del total ofertado, tiempo al primer turno libre) y tienden a ser desestimados por los equipos de gestión de Servicios de Salud.

\section{Conclusiones}

Consideramos que nuestro modelo de trabajo contribuyó a que en una gran proporción de pacientes que había consultado por dolor u otros síntomas generales, detectáramos, abordáramos y documentáramos el proceso de atención de problemas de la esfera psicosocial, que suele ser subregistrado con el abordaje biomédico clásico.

Conflicto de interés de los autores: No existen.

\section{Agradecimientos}

A Tessa Novick, Natalia Eugenia Pomari y Berenice Rabade, por su colaboración en la recolección de la información. A Paula Carrete, Judith Estremero, Adriana Goldman y Esteban Rubinstein, por los valiosos comentarios que hicieron a este escrito.

\section{Apéndice I. Guía explicativa para la realización de la ficha del dispositivo FOCO}

1. Queja explícita del paciente

Lo que el paciente percibe que le está sucediendo y trae a la consulta, usando sus mismas palabras. Puede estar expresado en el plano cognitivo, somático, emocional y/o de conducta.

2. ¿Por qué ahora?

Debe rastrearse específicamente qué hecho o secuencia de hechos tuvieron lugar para que la consulta surgiese en ese momento.

3. Relación con el contexto

Es importante conocer características del síntoma (dónde aparece, cuándo, cómo y cómo reaccionan las distintas personas involucradas). También es importante indagar factores atenuantes y perpetuadores del síntoma, creencias, temores y fantasías acerca del mismo, y de esta manera ayuda a discernir sobre distintos grados de estancamiento y afectación. ¿Qué interpreta el paciente que sucede? ¿Qué deja de hacer o quisiera hacer y no hace como consecuencia del problema?

4. Redefinición del Problema

EI MF, considerando los puntos anteriores, va construyendo con el paciente y/o familia un diagnóstico contextual operativo (DCO). Suele ocurrir que en dicho armado, el problema de consulta (astenia) se transforma en otro (depresión). A esto llamamos redefinición del problema, en el que el paciente llega con una queja o problema, y al ser evaluado por el médico y en esta construcción del DCO, se arriba a otro diagnóstico. Es importante que se trate de un problema "trabajable" (no son trabajables, por ejemplo, problemas económicos, o judiciales, pero sí sus consecuencias sobre la estructura familiar), con una meta alcanzable en un plazo de tiempo y observable en términos de conducta. También debe coincidir y ser coherente con lo que busca el paciente.

Algunos aspectos del problema a evaluar que nos proveen datos importantes para permitir el armado del DCO, son la relación con el contexto del paciente y las soluciones intentadas. De estas últimas es importante conocer si funcionaron o no, total o parcialmente. Es bueno reforzar aquellos intentos que funcionan o funcionaron alguna vez. Las soluciones intentadas que se hicieron y fracasaron 
aportan al mantenimiento del problema, dan una pauta rápida para saber por dónde no hay que ir. La intensidad con que intentaron manejar el problema nos da una medida del grado de afectación y de la importancia con que se considera al problema.

5. Motivación

- Referido al paciente: Qué busca al consultar y que esperaría de las entrevistas con el médico de familia. Qué está dispuesto a hacer. Qué expectativa tiene de esta serie de consultas.

- Referido al MF: Qué grado de influencia piensa que tiene sobre este paciente o esta familia. Esto quiere decir que la motivación se haga viable en la concreción de un objetivo con una táctica y una estrategia.

6. Objetivos

Elegir conjuntamente un aspecto del motivo de consulta a trabajar. Si es posible negociar una meta mínima a lograr a través de las entrevistas. Ejemplo: ¿Cómo se daría/n cuenta que está/n mejorando? ¿Cuál sería la mínima pauta/cambio que le/s haría saber que está/n yendo en la dirección correcta?

7. Plan para la próxima entrevista

Maniobras o modalidades de acción que el MF considera convenientes para el logro de su objetivo. Qué hacer, qué evitar hacer, a quiénes citar.

8. Notas

El médico tiene la posibilidad de escribir texto libre con datos que considere relevantes, situaciones o diálogos surgidos durante la consulta.

\section{Referencias}

1. Mcwhinney IR. Medicina de Familia. Madrid, Spain.: Mosby Doyma; 1994.

2. Epstein RM, Quill TE, Mcwhinney IR. Somatization reconsidered: incorporating the patient's experience of illness. Arch Intern Med . 1999;159(3):215222. Available from: 10.1001/archinte.159.3.215.

3. Allen J, Gay B, Crebolder H, Catholic JH, Svab I, Maastricht PR. The European definitions of the Key Features of the Discipline of General Practice, the role of the General Practitioner and a description of the Core Competencies of the General Practitioner / Family Physician. Evans P, editor. Barcelona, Spain; 2011. Available from: https://www.woncaeurope.org/sites/default/files/documents/Definition\%203rd\%20ed\%202011\% 20with\%20revised\%20wonca\%20tree.pdf

4. Huibers MJH, Beurskens A, Bleijenberg G, Schayck CPV. Psychosocial interventions by general practitioners. Cochrane Database Syst Rev. 2007;18(3):cd003494. Available from: 10.1002/14651858.cd003494.pub2.

5. Kok JLA, Williams A, Zhao L. Psychosocial interventions for people with diabetes and co-morbid depression. A systematic review. Int J Nurs Stud. 2015;52(10):1625-1639. Available from: 10.1016/j.ijnurstu.2015.05.012.

6. Rosendal M, Blankenstein AH, Morriss R, Fink P, Sharpe M, Burton C. Enhanced care by generalists for functional somatic symptoms and disorders in primary care. Cochrane Database Syst Rev. 2013;18(10):cd008142. Available from: 10.1002/14651858.cd008142.pub2.

7. Carrete P, Estremero J, Goldman A, Mutchinick M, Rubinstein E. FOCO. Familia, orientación y contexto. Profam texto y contexto. Servicio de Medicina Familiar y Comunitaria, Hospital Italiano de Buenos Aires. Buenos Aires, Argentina: delhospital Ediciones; 2016.

8. Comité Internacional de Clasificación de la WONCA. Clasificación Internacional de la Atención Primaria, segunda edición: CIAP-2. Barcelona, Spain: Masson; 1999. 